Editorial

\title{
The Underlying Mechanisms of Diabetic Myopathy
}

\author{
Erick O. Hernández-Ochoa, ${ }^{1}$ Paola Llanos, ${ }^{2}$ and Johanna T. Lanner ${ }^{3}$ \\ ${ }^{1}$ Department of Biochemistry and Molecular Biology, University of Maryland School of Medicine, Baltimore, USA \\ ${ }^{2}$ Institute for Research in Dental Sciences, Facultad de Odontología, Universidad de Chile, Santiago, Chile \\ ${ }^{3}$ Department of Physiology and Pharmacology, Karolinska Institutet, Stockholm, Sweden
}

Correspondence should be addressed to Erick O. Hernández-Ochoa; ehernandez-ochoa@som.umaryland.edu

Received 2 July 2017; Accepted 4 July 2017; Published 7 November 2017

Copyright (C) 2017 Erick O. Hernández-Ochoa et al. This is an open access article distributed under the Creative Commons Attribution License, which permits unrestricted use, distribution, and reproduction in any medium, provided the original work is properly cited.

\section{Editorial}

Late complications of diabetes affect both the quality and quantity of life, resulting in major health costs [1]. A common complication of both type 1 diabetes (T1D) and type 2 diabetes (T2D) is the failure to preserve muscle mass and function [2, 3], here referred to as diabetic myopathy [4-6]. Although often overlooked, this complication is believed to contribute to the progression of other diabetic complications and comorbidities (e.g., uncontrolled hyperglycemia, sedentarism, and obesity) based on the key role skeletal muscle plays in glucose homeostasis and locomotion [7-9]. Numerous studies have investigated the link between diabetic myopathy and diverse cellular processes [10]; however, despite the wealth of information on muscle weakness and muscle wasting [11], the specific triggering events of diabetic myopathy in patients with diabetes remain unknown. Further knowledge of the pathophysiological and molecular mechanisms involved in the onset and progression of diabetic myopathy is needed for the development of new pharmacological tools to ameliorate diabetic myopathy. The importance of this area of diabetes research was the motivation for us to develop this special issue.

This special issue includes three review articles and two original research papers, from leading and emerging scientists who study diabetic myopathy in different muscle tissues (cardiac, smooth, and skeletal) and with diverse expertise and interests, aiming to stimulate the continuing effort to understand the impact of diabetes on muscle function. These reviews and research papers represent the joint effort of 23 experts in the field, in which they examine the topic from several angles and levels ranging from translational aspects to whole tissue and single cell.

G. Barrientos and collaborators present a review about membrane cholesterol in skeletal muscle and its role in excitation-contraction coupling and glucose transport. They focus on the muscle plasma membrane network characterized by surface invaginations, also known as the transverse tubular (t-tubular) system [12]. This t-tubular system is rich in cholesterol [13], and it is critical for excitability and bidirectional transport of solutes, ions, nutrients, and metabolic waste. The authors discuss recent findings by other groups [14] and the work that they have done, regarding t-tubular cholesterol dynamics and its effects on excitationcontraction coupling and GLUT4 trafficking in normal and obese animal models $[15,16]$. In obesity, the cholesterol content in the t-tubular network is further increased [16]. Interestingly, as suggested by the authors, restoring cholesterol levels and increasing GLUT4 trafficking in the t-tubular system could represent a new therapeutic avenue to ameliorate insulin resistance.

P. E. Morales et al. review the topic of muscle lipid metabolism and the role of lipid droplets and perilipins in rodents and humans. The skeletal muscle is not only important for carbohydrate metabolism, it is also crucial for the metabolism of lipids. Obesity is characterized by aberrant fat storage and increased levels of circulating lipids and fatty acids [17]. Lipotoxicity is characterized by the uncontrolled intracellular accumulation of lipids, oxidative stress, organelle damage, and autophagy [18]. Lipid droplets are intracellular depots, 
limited by phospholipid monolayer, and represent an important an immediate source of energy substrates [19]. Lipid droplets are localized mainly in the subsarcolemmal region or in between myofibrils abutted to mitochondria [20]. Here, the authors present insights into the mechanisms underlying lipid trafficking and its metabolism in skeletal muscle, especially focusing on the function of lipid droplets, the PLIN family of proteins and how these entities are modified during mechanical contraction, obesity, and insulin resistance.

The review by $\mathrm{D}$. T. Au et al. focuses on the $\mathrm{LDL}$ receptor-related protein 1 (LRP1), a signaling receptor member of the low-density lipoprotein (LDL) receptor family involved in the clearance of chylomicrons from the circulation [21]. LRP1 is widely expressed in different tissues, with high expression in hepatocytes, adipocytes, fibroblasts, macrophages, and vascular smooth muscle cells, where it plays a critical role during angiogenesis and as an atheroprotective factor (i.e., protects against the formation of atherosclerosis) [22]. D. T. Au et al. discuss recent results using liver-specific LRP1 knockout mice [23]. Hepatic LRP1 inactivation resulted in defective insulin signaling, which included impaired phosphorylation of insulin receptor. These authors also summarize recent advances in LRP1 function in adipocytes, including studies using adipocyte LPR1 knockout mice that displayed delayed postprandial lipid clearance and improved glucose tolerance and resistance to high-fat dietinduced obesity [24]. The authors also highlight recent evidence in support of a link between LPR1 from epicardial adipocytes and glucose metabolism in individuals with T2D [25]. Due to the close proximity to muscle cells, intra- and intermuscular adipocytes may communicate with myofibers from skeletal, smooth, and cardiac tissues. Future work on LRP1 signaling could further our understanding of the cross talk between intramuscular adipocytes and the muscle.

E. O. Hernández-Ochoa and colleagues' contribution to this special issue concerns altered action potential-induced $\mathrm{Ca}^{2+}$ transients in cultured skeletal muscle fibers challenged with elevated extracellular glucose. As mentioned, patients with T1D and T2D exhibit increased muscle weakness and loss of muscle mass $[2,3,11]$. Yet, the mechanisms underlying diabetic myopathy remain unknown. Previous studies in skeletal muscle reported alterations in the excitationcontraction coupling (ECC) - a coordinated chain of cellular events that links the membrane action potential with intracellular $\mathrm{Ca}^{2+}$ release and activation of the contractile machinery $[26,27]$. This study shows that muscle fibers cultured in elevated glucose for $48 \mathrm{hrs}$ exhibit predominantly biphasic action potential-induced $\mathrm{Ca}^{2+}$ transients in response to single field stimulation. Thus, glucose-induced alterations in $\mathrm{Ca}^{2+}$ transients could play a role in the progression of muscle weakness and observed in diabetic myopathy.

The paper by M. L. Mizgier et al. investigated the effect of human myotube-derived media on glucose-stimulated insulin secretion in mice pancreatic islets and rat-isolated beta cells. The glucose homeostasis and insulin secretion are tightly regulated in our body [7-9]. The skeletal muscle accounts for $70-80 \%$ of the insulin-dependent glucose disposal in the postprandial state in humans [7]. The skeletal muscle is supposedly also involved in the regulation of insulin secretion from the pancreas via muscle humoral factors, also known as myokines [28, 29]. In this study, they monitored five myokines: IL6, IL8/CXCL8, MCP1/CCL2, fractalkine/CX3CL1, and RANTES/CCL5. They hypothesized that insulin influences the secretion of myokines, which in turn increases the glucose-stimulated insulin secretion (GSIS). Pancreatic islets incubated with conditioned media from human myotubes exposed to glucose exhibited higher GSIS than islets exposed to conditioned media from human myotubes in the absence of glucose stimulation. Furthermore, conditioned media from insulin-treated myotubes did not influence GSIS. They conclude that myokines present in conditioned media from untreated human myotubes regulate insulin secretion in mice pancreatic islets. These results support their hypothesis that myokines can influence insulin secretion.

We hope that more of our colleagues become interested in the study of diabetic myopathy and contribute to further our knowledge of the mechanisms of this understudied pathological process.

\section{Acknowledgments}

We would like to thank all the authors and editors for their contributions to this special issue. Special thanks to the external reviewers who contributed their expertise, evaluated the manuscripts, and provided useful criticisms. Support for the preparation of this editorial was provided by the National Institute of Arthritis and Musculoskeletal and Skin Diseases (NIH) R37-AR055099 (Erick O. Hernández-Ochoa); FONDECYT11150243, CONICYT-Chile, and FIOUCh-Enlace 001/2015, Facultad de Odontología, Universidad de Chile (Paola Llanos); and Swedish Research Council, Magnus Bergvall Stiftelse, and Åke Wiberg Stiftelse (Johanna T. Lanner).

\section{Erick O. Hernández-Ochoa Paola Llanos \\ Johanna T. Lanner}

\section{References}

[1] J. M. Forbes and M. E. Cooper, "Mechanisms of diabetic complications," Physiological Reviews, vol. 93, no. 1, pp. 137-188, 2013.

[2] S. W. Park, B. H. Goodpaster, J. S. Lee et al., "Excessive loss of skeletal muscle mass in older adults with type 2 diabetes," Diabetes Care, vol. 32, no. 11, pp. 1993-1997, 2009.

[3] S. W. Park, B. H. Goodpaster, E. S. Strotmeyer et al., "Decreased muscle strength and quality in older adults with type 2 diabetes: the health, aging, and body composition study," Diabetes, vol. 55, no. 6, pp. 1813-1818, 2006.

[4] H. Andersen, P. C. Gadeberg, B. Brock, and J. Jakobsen, "Muscular atrophy in diabetic neuropathy: a stereological magnetic resonance imaging study," Diabetologia, vol. 40, no. 9, pp. 1062-9, 1997.

[5] M. P. Krause, M. C. Riddell, C. S. Gordon, S. A. Imam, E. Cafarelli, and T. J. Hawke, "Diabetic myopathy differs between Ins $2^{\text {Akita+/- }}$ and streptozotocin-induced type 1 diabetic models," Journal of Applied Physiology, vol. 106, no. 5, pp. 1650-1659, 2009. 
[6] E. O. Hernandez-OchoaC. Vanegas, "Diabetic myopathy and mechanisms of disease," Biochemistry \& Pharmacology: Open Access, vol. 4, no. 05, p. 2015.

[7] E. Ferrannini, O. Bjorkman, G. A. Reichard Jr et al., "The disposal of an oral glucose load in healthy subjects. A quantitative study," Diabetes, vol. 34, no. 6, pp. 580-588, 1985.

[8] E. Ferrannini, D. C. Simonson, L. D. Katz et al., "The disposal of an oral glucose load in patients with non-insulin-dependent diabetes," Metabolism, vol. 37, no. 1, pp. 79-85, 1988.

[9] B. B. Kahn, A. S. Rosen, J. F. Bak et al., "Expression of GLUT1 and GLUT4 glucose transporters in skeletal muscle of humans with insulin-dependent diabetes mellitus: regulatory effects of metabolic factors," The Journal of Clinical Endocrinology \& Metabolism, vol. 74, no. 5, pp. 1101-1109, 1992.

[10] D. M. D'souza, D. Al-Sajee, and T. J. Hawke, "Diabetic myopathy: impact of diabetes mellitus on skeletal muscle progenitor cells," Frontiers in Physiology, vol. 4, p. 4, 2013.

[11] H. Andersen, O. Schmitz, and A. S. Nielsen, "Decreased isometric muscle strength after acute hyperglycaemia in type 1 diabetic patients," Diabetic Medicine, vol. 22, no. 10, pp. 1401-1407, 2005.

[12] C. Franzini-Armstrong and L. D. Peachey, "Striated musclecontractile and control mechanisms," The Journal of Cell Biology, vol. 91, no. 3, pp. 166s-1186, 1981.

[13] M. Rosemblatt, C. Hidalgo, C. Vergara, and N. Ikemoto, "Immunological and biochemical properties of transverse tubule membranes isolated from rabbit skeletal muscle," Journal of Biological Chemistry, vol. 256, no. 15, pp. 8140-8148, 1981.

[14] M. Z. Haque, M. I. VJ, A. B. Abou Samra, R. M. Mohammad, and R. D. Lasley, "Cholesterol depletion alters cardiomyocyte subcellular signaling and increases contractility," PLoS One, vol. 11, no. 7, article E0154151, 2016.

[15] G. Barrientos, P. Llanos, J. Hidalgo et al., "Cholesterol removal from adult skeletal muscle impairs excitation-contraction coupling and aging reduces caveolin-3 and alters the expression of other triadic proteins," Frontiers in Physiology, vol. 6, p. 105, 2015.

[16] P. Llanos, A. Contreras-Ferrat, T. Georgiev et al., "The cholesterol-lowering agent methyl- $\beta$-cyclodextrin promotes glucose uptake via GLUT4 in adult muscle fibers and reduces insulin resistance in obese mice," American Journal of Physiology Endocrinology and Metabolism, vol. 308, no. 4, pp. E294-E305, 2015.

[17] J. Roth, X. Qiang, S. L. Marbán, H. Redelt, and B. C. Lowell, "The obesity pandemic: where have we been and where are we going?," Obesity Research, vol. 12, no. S11, pp. 88s-101s, 2004.

[18] C. Brons and L. G. Grunnet, "Mechanisms in endocrinology: skeletal muscle lipotoxicity in insulin resistance and type 2 diabetes: a causal mechanism or an innocent bystander?," European Journal of Endocrinology, vol. 176, no. 2, pp. R67-R78, 2017.

[19] C. W. Wang, "Lipid droplets, lipophagy, and beyond," Biochimica et Biophysica Acta (BBA) - Molecular and Cell Biology of Lipids, vol. 1861, no. 8, Part B, pp. 793-805, 2016.

[20] M. Bosma, "Lipid droplet dynamics in skeletal muscle," Experimental Cell Research, vol. 340, no. 2, pp. 180-186, 2016.

[21] A. P. Lillis, L. B. Van Duyn, J. E. Murphy-Ullrich, and D. K. Strickland, "Ldl receptor-related protein 1: unique tissue- specific functions revealed by selective gene knockout studies," Physiological Reviews, vol. 88, no. 3, pp. 887-918, 2008.

[22] D. K. Strickland, D. T. Au, P. Cunfer, and S. C. Muratoglu, "Low-density lipoprotein receptor-related protein-1: role in the regulation of vascular integrity," Arteriosclerosis, Thrombosis, and Vascular Biology, vol. 34, no. 3, pp. 487-498, 2014.

[23] Y. Ding, X. Xian, W. L. Holland, S. Tsai, and J. Herz, "Lowdensity lipoprotein receptor-related protein-1 protects against hepatic insulin resistance and hepatic steatosis," eBioMedicine, vol. 7, pp. 135-145, 2016.

[24] S. M. Hofmann, L. Zhou, D. Perez-Tilve et al., "Adipocyte Ldl receptor-related protein-1 expression modulates postprandial lipid transport and glucose homeostasis in mice," Journal of Clinical Investigation, vol. 117, no. 11, pp. 3271-3282, 2007.

[25] L. Nasarre, O. Juan-Babot, P. Gastelurrutia et al., "Low density lipoprotein receptor-related protein 1 is upregulated in epicardial fat from type 2 diabetes mellitus patients and correlates with glucose and triglyceride plasma levels," Acta Diabetologica, vol. 51, no. 1, pp. 23-30, 2014.

[26] K. G. Beam And and P. Horowicz, "Excitation-contraction coupling in skeletal muscle," in Myology, vol. 1p. 2004.

[27] M. F. SchneiderE. O. Hernandez-Ochoa, Skeletal Muscle Excitation-Contraction Coupling in Muscle: Fundamental Biology and Mechanisms of Disease, J. A. Hill and , Eds., Academic Press, Elsevier, London, Uk, 2012.

[28] K. Bouzakri, P. Plomgaard, T. Berney, M. Y. Donath, B. K. Pedersen, and P. A. Halban, "Bimodal effect on pancreatic $\beta$-cells of secretory products from normal or insulinresistant human skeletal muscle," Diabetes, vol. 60 , no. 4, pp. 1111-1121, 2011.

[29] H. Ellingsgaard, I. Hauselmann, B. Schuler et al., "Interleukin6 enhances insulin secretion by increasing glucagon-like peptide-1 secretion from L cells and alpha cells," Nature Medicine, vol. 17, no. 11, pp. 1481-1489, 2011. 


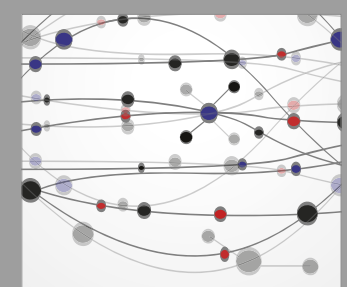

The Scientific World Journal
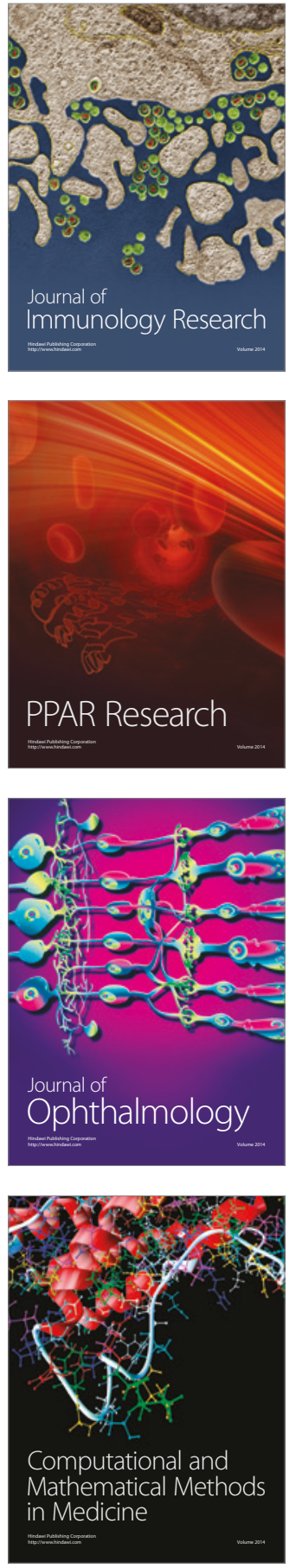

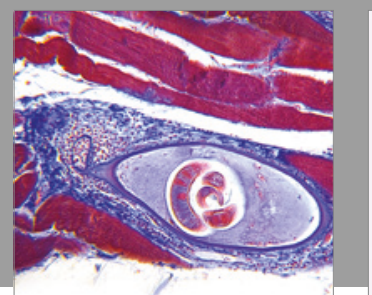

Gastroenterology Research and Practice
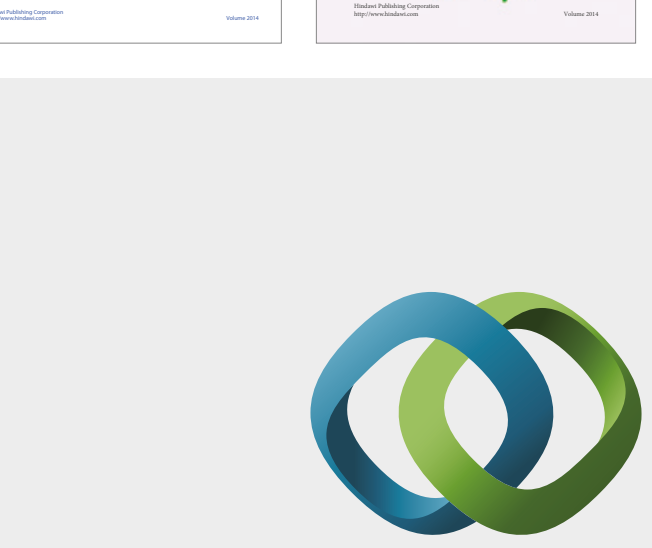

\section{Hindawi}

Submit your manuscripts at

https://www.hindawi.com
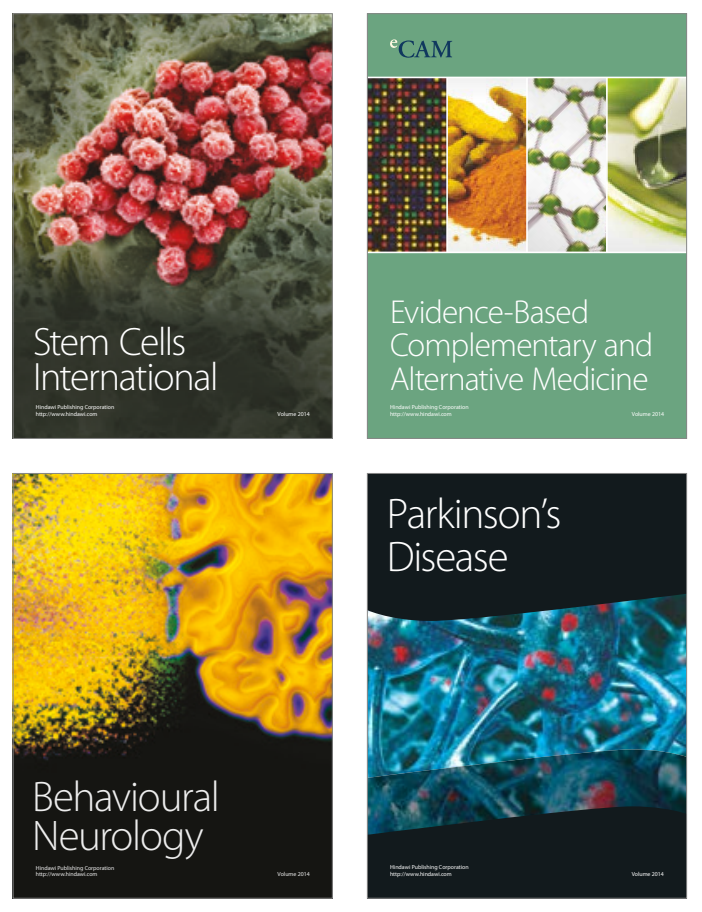
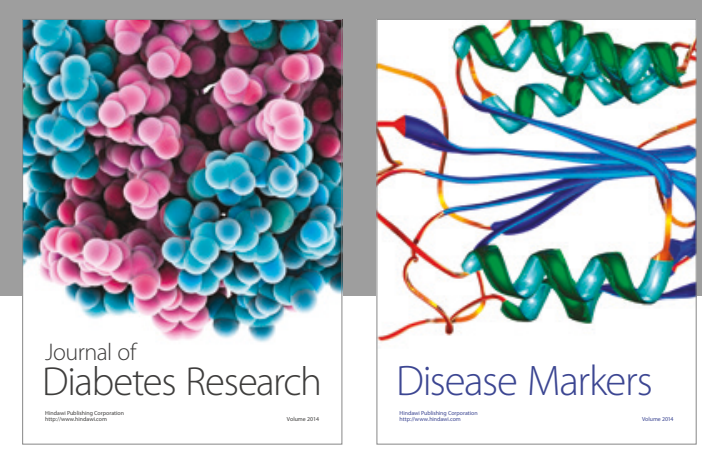

Disease Markers
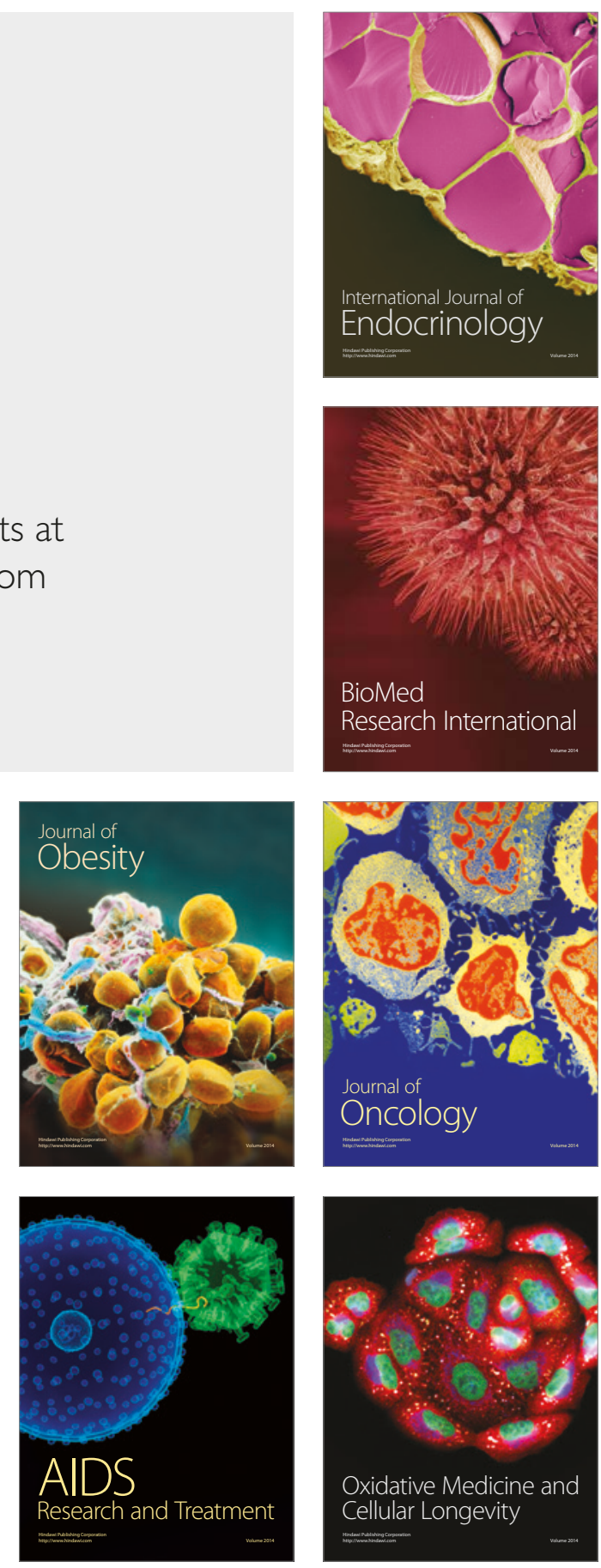